\title{
Research on Canal System Operation Based on Controlled Volume Method
}

\author{
Zhiliang Ding \\ Changjiang Institute of Survey, Planning, Design and Research, Wuhan 430010, China \\ Email: dingzhiliang@126.com \\ Changde Wang \\ State Key Laboratory of Water Resources and Hydropower Engineering Science, Wuhan University, \\ Wuhan, 430072, China \\ Email:cdwwh@tom.com
}

\begin{abstract}
An operating simulation mode based on storage volume control method for multireach canal system in series was established. In allusion to the deficiency of existing controlled volume algorithm, the improved algorithm was proposed, that is the controlled volume algorithm of whole canal pools, the simulation results indicate that the storage volume and water level of each canal pool can be accurately controlled after the improved algorithm was adopted. However, for some typical discharge demand change operating conditions of canal, if the controlled volume algorithm of whole canal pool is still adopted, then it certainly will cause some unnecessary regulation, and consequently increases the disturbed canal reaches. Therefor, the idea of controlled volume operation method of continuous canal pools was proposed, and its algorithm was designed. Through simulation to practical project, the results indicate that the new controlled volume algorithm proposed for typical operating condition can comparatively obviously reduce the number of regulated check gates and disturbed canal pools for some typical discharge demand change operating conditions of canal, thus the control efficiency of canal system was improved. The controlled volume method of operation is specially suitable for large-scale water delivery canal system which possesses complex operation requirements.
\end{abstract}

Index Terms - canal operation, controlled volume method, control algorithm, simulation

\section{INTRODUCTION}

A canal system can be operated by managing the water volume contained in one or more canal pools, and the volume can be changed to satisfy operational criteria by allowing the pivot point to move within each pool, this is the controlled volume method of operation [1]. The controlled volume method of operation offers the most flexibility of any method of operation. Because a constant depth limitation does not exist, the operational flexibility primarily is restricted by depth fluctuation limits. A canal

Footnotes: 8-point Times New Roman font;

Manuscript received January 1, 2008; revised June 1, 2008; accepted July 1, 2008.

Copyright credit, project number, corresponding author, etc. system operating by the controlled volume method of operation has the capability to respond to a wide range of flow conditions. Depth fluctuation can be managed to avoid rapid drawdown at the upstream ends of the pools without wasting water or exceeding the maximum depth allowed at the downstream ends. Another aspect might be to transform rapid flow change at the downstream end into a gradual flow change in the upstream canal pools by using pool storage as a buffer, and as a result, the disturbance to the upper pools is minimized.

The controlled volume method of operation requires using the supervisory control method and the complex software developed according to local specific conditions, so this method of operation is most difficult to bring into effect. Although there are many successful engineering examples to adopt the controlled volume method of operation in overseas canal systems [2]-[5], but the control algorithms can only be suitable for their specific operation plans, also there is not detailed data introduction for their concrete methods. Yao et al. [6]-[7] established the operation mode of serial canal system based on the controlled volume method, and the model was successfully tested in the testing canal system proposed by ASCE, but he has not made detailed introduction for specific algorithm to realize the volume control process, also the accurate volume control is not realized. A brief and effective controlled volume algorithm is proposed in the paper on the foundation of predecessors's studies, and the simulation is carried out to the practical project.

\section{OPERATIONAL CONTROL MODEL OF CANAL SYSTEM}

\section{A. Mathematical Model of Canal System}

Usually the main canal is divided into canal pools by check gates in series which contains some canalside turnouts. The check gates of upstream and downstream are boundaries of the conjoint pools. The water transfer canal system showed in Fig. 1 is composed of $n$ canal pools, and there is a huge reservoir upstream as water source [1]. 


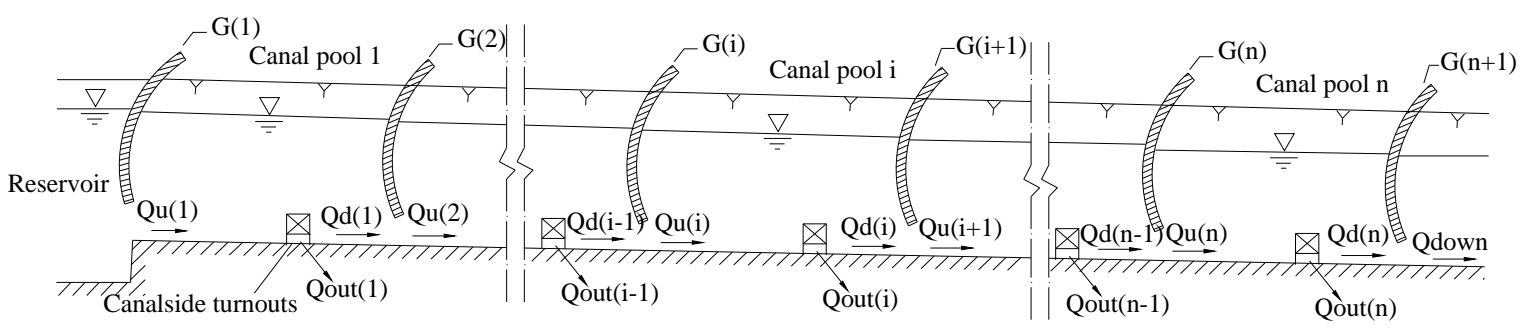

Figure 1. Schematic diagram of a canal system

The unsteady dynamic characteristic of channel can be described by Saint-Venant equations, which are composed by equation of continuity and equation of momentum [8]-[9]:

$$
\begin{gathered}
B \frac{\partial Z}{\partial t}+\frac{\partial Q}{\partial s}=q \\
\frac{1}{g A} \frac{\partial Q}{\partial t}+\frac{2 Q}{g A^{2}} \frac{\partial Q}{\partial s}+\left(1-\frac{B Q^{2}}{g A^{3}}\right) \frac{\partial Z}{\partial s}= \\
\frac{q}{g A}\left(v_{q s}-v\right)+\frac{B Q^{2}}{g A^{3}}(i+M)-\frac{Q^{2}}{A^{2} C^{2} R}
\end{gathered}
$$

Where $B$ is the width of canal water surface, $Z$ is the water surface elevation, $t$ is the time, $Q$ is the discharge, $C$ is the Chezy coefficient, $S$ is the distance along the canal, $g$ is the gravitational acceleration, $A$ is the area of cross section, $q$ is the inflow along the canalside, $v$ is the flow velocity along canal axes, $R$ is the hydraulics radius, $v_{q s}$ is the average flow velocity along canal axes of the canalside inflow, $i$ is the canal bottom slope, $M=\left.\frac{1}{B} \frac{\partial A}{\partial s}\right|_{h}$, for prism shape canal, $M=0$.

The preissmann implicit scheme is widely adopted as the numerical solution method of Saint-Venant equations for its fine characteristics such as high accuracy, unconditional convergence [10].

\section{B. Simulation Model of Canal System}

\section{1) Frame of control system}

The scheduled control targets are realized by adjusting the control equipment, such as the check gate according to the required pool water level and flow along the canal. And both the hydraulic characteristics and the application of control theory associated deserve consideration in the canal system of automatic control.

The discharge feedforward plus water level feedback PI controller is proposed in simulation model, the frame of the control system is presented in Fig. 2. The entire canal automation simulation system is composed by six modules: the steady flow calculation module, the gate opening inverse- solution module, the PI control module, the calculation module for gate opening, the calculation module for the discharge through the gate and the unsteady flow calculation module. These six modules contact hermetically and interact to realize canal operation according designed operation method, and satisfy downstream discharge demand.

\section{2) Design of controller}

The controller adopts the combinative mode of the feed forward and feedback. The discharge feed forward and water level feedback controllers are designed respectively to regulate the gate openings together. The output of the entire controller is the overlying gate opening variation value. Of course, it can not be final input of the gate hoist device until it satisfies the restrictions of the gate deadband and maximum gate moving speed.

Feed forward control of discharge: according to the preallocated discharge of gate acquired from the controlled volume algorithm in (9)-(12), and the upstream and downstream water levels of gates of the last calculation period computed by the Saint-Venant equations, the gate opening $G_{k}$ can be derived reversely from the gate discharge calculation formula. Then $G_{k}$ is compared with its value of last calculation period, and the increment $\Delta G_{1}$ of gate opening is acquired.

$$
Q=C_{d} G_{k} b \sqrt{2 g h_{0}}
$$

Where $Q$ is the discharge through the gate, $C_{d}$ is the discharge coefficient, $G_{k}$ is the gate opening, $b$ is the gate width, $h_{0}$ is the upstream water depth.

Feed back control of water level: according to the real-time target water level $Y T$ and the feedback value $Y F$ of target water level derived from the unsteady flow calculation, the increment $\Delta G_{2}$ of gate opening is acquired through the incremental PI control algorithm:

$$
\Delta G_{2}=K_{P} \Delta e(j)+K_{I} e(j)
$$

Where $\Delta G_{2}$ is the output of feedback control, $K_{P}$ is the proportional coefficient, $K_{I}$ is the integral coefficient, $e$ is the difference of water levels, $\Delta e$ is described by $\Delta e(j)=e(j)-e(j-1), j$ is the sampling time.

\section{ALGORITHM DESIGN OF THE CONTROLLED VOLUME OPERATION OF WHOLE CANAL POOLS}

Under the controlled volume method of operation, the water level in canal may rise and fall. But in order to avoid water overtopping the freeboard and satisfy the demand of canalside water diversion, it must satisfy certain water level restriction conditions. With respect to 


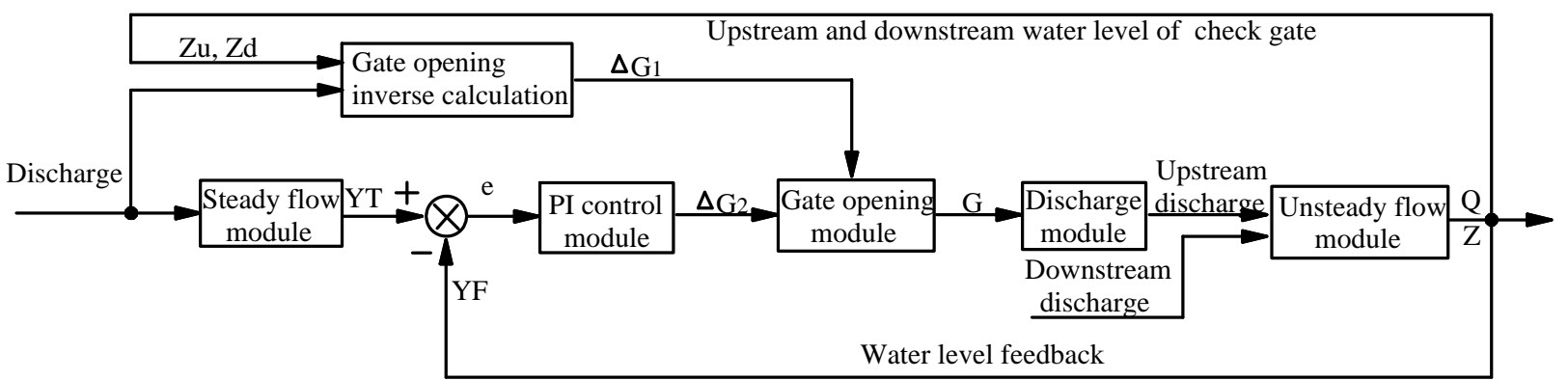

Figure 2. Canal automation control system

multireach serial canal system, under the condition of certain flow discharge, each canal reach should have its minimum restrained water level $Z_{\text {min }}$ and maximal restrained water level $Z_{\max }$. The target water level $Y T$ varies with the variation of demanding discharge at canalside turnouts and canal downstream end in the control process and it is not a constant value. The controlled volume method of operation proposed in the paper adopts the real-time midpoint-weighted value of water level of canal reach upstream and downstream end, as the real-time target water level of the water level feedback control. Correspondingly with this, the initial target water level subtracts and adds on an allowable water level amplitude of variation, namely it is the minimum restrained water level $Z_{\text {min }}$ and maximal restrained water level $Z_{\max }$ of the controlled volume operation. The allowable water level amplitude of variation can be properly selected according to the canalside turnout constraint and the canal freeboard constraint. From analysis we can see that, different canal pool volume corresponds to the different midpoint-weighted water level of this canal pool. Therefore, the variation range of canal pool storage volume of controlled volume operation is situated through the setup of different limiting water level of canal pool.

When the most downstream end of canal has discharge demand variation, then the controlled volume operation regulation needs be carried through to the whole canal, which is the controlled volume operation of whole canal pools.

\section{A. Regulation of Discharge Difference}

Supposes the initial total demand of discharge for the entire canal system is:

$$
Q_{\text {con }}=Q_{d o}+\sum_{i=1}^{n} Q_{o}(i)
$$

The total demand of discharge in other time for canal system is:

$$
Q_{\text {sum }}=Q_{\text {down }}+\sum_{i=1}^{n} Q_{\text {out }}(i)
$$

Then the variation of the total demand of discharge, namely the discharge difference is:

$$
Q_{\text {lack }}=Q_{\text {sum }}-Q_{\text {con }}
$$

Where $Q_{d o}$ is the water taking discharge at the canal downstream end under initial state, $Q_{o}(i)$ is the water diversion discharge at each canalside turnout under initial state. $Q_{\text {down }}$ and $Q_{\text {out }}(i)$ are the water taking discharge at the canal downstream end and the water diversion discharge at each canalside turnout in other time, respectively. Actually, each canal pool may have more than one turnout, in order to be abbreviated expression, $Q_{\text {out }}(i)$ represents the total water taking discharge in canal pool $i(i=1,2 \ldots, n-1, n)$.

Then the discharge difference between the inflow and outflow in each canal pool is:

$$
q_{\text {lack }}^{j}(i)=R_{t} \times Q_{\text {lack }}^{j} \times \frac{V^{j}(i)}{\sum_{i=1}^{n} V^{j}(i)}
$$

Where $q_{\text {lack }}{ }^{j}(i)$ is the assigned discharge difference of canal pool $i$ in the time $j, Q_{\text {lack }}^{j}=Q_{\text {sum }}{ }^{j}-Q_{\text {con }}$, and $Q_{\text {lack }}^{j}$ is the discharge difference between the real-time total demand of discharge and its initial value. $V^{j}(i)$ is the storage volume of canal pool $i$ in the time $j . R_{t}$ is the weighting coefficient of discharge difference in the first round storage volume regulation, $R_{t} \in(0,1]$, and it determines directly the strong or weak degree of the volume regulation, and enables the rulers to control the volume regulation speed according to requirements.

From the downstream end canal pool $n$ to the upstream canal pool $i$ ( $i=n-1, n-2, \cdots 2,1)$, the upstream and downstream gate discharge of each canal pool are in turn:

$$
\begin{gathered}
Q_{u}(n)=Q_{\text {out }}(n)+Q_{\text {down }}-q_{\text {lack }}(n) \\
Q_{d}(n)=Q_{\text {down }} \\
Q_{u}(i)=Q_{\text {out }}(i)+Q_{u}(i+1)-q_{\text {lack }}(i) \\
Q_{d}(i)=Q_{u}(i+1)
\end{gathered}
$$

The upstream and downstream gate discharge of each canal pool acquired through the controlled volume algorithm is namely the input variable of discharge feed 
forward in the controller of canal system.

\section{B. Real-time Target Water Level Calcalation}

The water volume is a variable under the controlled volume method of operation, and the flow state in canal pool varies with the variation of discharge demand at the canal downstream end and canalside turnouts. According to the discharge difference of each canal pool, within the sampling period $j$, the variation of water volume for canal pool $i$ is:

$$
D V^{j}(i)=q_{\text {lack }}^{j}(i) \times D T
$$

Then the new water volume of canal pool $i$ is:

$$
V C^{j}(i)=V C^{j-1}(i)+D V^{j}(i)
$$

Where $V C^{j-1}(i)$ is the water volume of the last calculation period of canal pool, if the last calculation period is the initial time, then $V C^{j-1}(i)=V_{0}(i), D T$ is the calculation period.

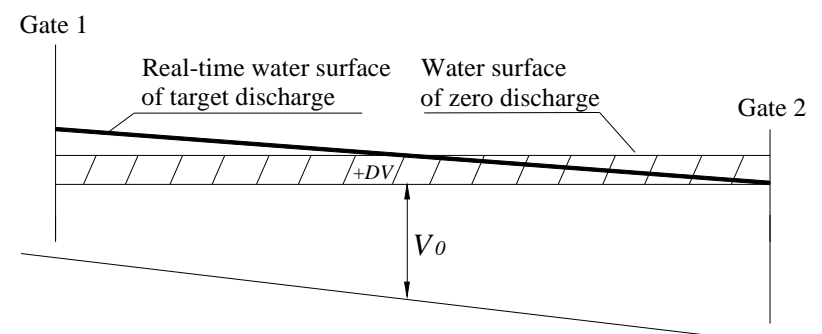

Figure 3. Real-time water surface profile

When there is new water volume of each canal pool, the water surface profile position of zero flow rate can be determined under this water volume. And then according to the target discharge state of corresponding time determined by discharge difference dispatching, then the singular target water surface profile of this time is determined. In the process of computer control operation, the real-time midpoint-weighted water level can be ascertained according to following method: firstly, supposes a downstream end water level of a canan pool, then uses the steady flow calculation module to calculate the water surface profile, and then the canal pool volume correspondingly to this supposed water level can be acquired, and the comparison is made between this volume with the real-time target volume computed by (14). If both are equal, then the supposed downstream water level is correct, otherwise, supposes the downstream end water level and calculates corresponding canal pool volume over again until the result is equal to the real-time target volume of this time. We can use the dichotomy method to carry on above iterative calculation.

When there is new real-time target water level and the real-time water level information derived from the unsteady flow calculation, the real-time water level difference can be acquired, and then it is regarded as the input variable of feedback controller to perform water level feedback control.

\section{Determination of the Target Water Level and Restricted Water Level}

Both the target water level and target water volume of each canal pool under controlled volume operation can be regarded as the known values. Supposes the target water level of each canal pool is $Y T_{\text {tar }}(i)$ when canal system stabilizes, and the target water volume of each canal poo is $V_{\text {tar }}(i)$. The restricted water levels of each canal pool are $Z_{\text {min }}(i)$ and (or) $Z_{\max }$ (i) when the flow equilibrium regulation begins. Fig. 4 shows the linear reversion process of discharge difference, in which $T_{j}$ is the time to begin the second round regulation, and the discharge difference between the inflow and outflow in each canal pool at this time is $q_{\text {lack }}(i), T_{s}$ is the time to achieve equilibrium between the inflow and outflow. The time needed for linear subduction of discharge difference can be properly selected according to the stable requirement of system control. Usually the larger the discharge difference $q_{\text {lack }}(i)$ is, the higher requirement of system stability is in the waterpower transient process of canal system, and the longer linear recovery time of discharge difference is needed.

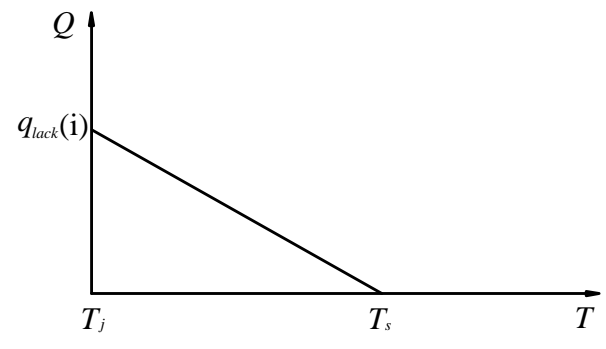

Figure 4. Restoration of discharge balance

Under the flow equilibrium regulation stage, the variation of water volume in each canal pool is:

$$
\Delta V(i)=\frac{1}{2} \times q_{\text {lack }}(i) \times\left(T_{s}-T_{j}\right)
$$

Therefore, at the time when the flow equilibrium regulation begins, the water volume of each canal pool is:

$$
V(i)=V_{\text {tar }}(i)-\Delta V(i)
$$

Author's study indicates the difference of the time to begin the flow equilibrium regulation for each canal pool is correspondingly small, and the discharge difference assigned to each canal pool is correspondingly smaller than the upstream gate discharge of this canal pool, so the upstream and downstream gate discharge of each canal pool from downstream end to upstream of canal system can be expressed approximately by (9)-(12) in turn when the flow equilibrium regulation begins.

Supposes when the flow equilibrium regulation begins, the discharge variation at each canalside turnout and canal downstream end has been stable, obviously, the discharge at upstream and downstream boundaries of each canal pool and each canalside turnout is known in this time. Because the beginning time of the flow 
equilibrium regulation for each canal pool may not be same, so there is certain difference between the actual rate flow and its calculation value at the upstream and downstream boundaries when the flow equilibrium regulation begins for each canal pool, but simulation indicates such difference is correspondingly small, and its influence on the target water level (or target water volume) calculation is very small, so the difference can be ignored

Before the simulation begins, according to the known water volume and discharge conditions of each canal pool when the flow equilibrium regulation begins, we can use the steady flow calculation module to calculate the singular water surface profile of each canal pool of this time, then the midpoint-weighted water level calculated from the upstream and downstream end water levels of this water surface profile is namely the restricted water level $Z_{\min }(i)$ or $Z_{\max }(i)$ of each canal pool when the flow equilibrium regulation begins.

\section{Flow equilibrium regulation algorithm}

When the real-time water volume of canal pool approaches the target volume $V T(i)$, the canal system needs to carry out the second round regulation to make the inflow and outflow of canal pool balanceable. Suppose the upstream gate flow rate of each canal pool is $Q_{u}(i)$ in the flow equilibrium regulation stage, the flow rate difference is $q_{\text {lack-t }}(i)$ when the flow equilibrium regulation begins, and the simulation period is $D T$. Then the calculation period times needed for accomplishing the flow equilibrium regulation of each canal pool is:

$$
K_{n}(i)=\frac{T_{s}-T_{j}}{D T}
$$

Where $K_{n}(i)$ is the calculation period times needed to accomplish the flow equilibrium regulation of each canal pool; the meanings of other symbols are the same as the former.

Supposes the real-time calculation period number of each pool is $K^{j}(i)$ at the flow equilibrium regulation stage, and the real-time recovery quantity of the flow rate difference of each pool is $Q_{\text {sup }}(i)$, then $K^{j}(i)=0$ and $Q_{\text {sup }}(i)=0$ in canal pools without flow equilibrium regulation, and $1 \leq K^{j}(i) \leq K_{n}(i)$ in canal pools without flow equilibrium regulation.

If the canal pool has performed the flow equilibrium regulation, then its real-time recovery quantity of the flow rate difference is:

$$
\begin{aligned}
& \text { If } 1 \leq K^{j}(i) \leq K_{n}(i) \\
& \text { then } Q_{\text {sup }}(i)=\frac{K^{j}(i)}{K_{n}(i)} \times q_{\text {lack-t }}(i) \\
& \text { If } K^{j}(i)>K_{n}(i)
\end{aligned}
$$

$$
\text { then } Q_{\text {sup }}(i)=q_{\text {lack-t }}(i)
$$

At the flow equilibrium regulation stage, from the downstream end canal pool $n$ to the upstream canal pool $i(i=n-1, n-2, \ldots 2,1)$, the upstream and downstream gate discharge of each canal pool is in turn (all variables have abbreviated the time coordinates for simplification ):

$$
\begin{gathered}
Q_{u}(n)=Q_{\text {out }}(n)+Q_{\text {down }}-q_{\text {lack-t }}(n)+Q_{\text {sup }}(n) \\
Q_{d}(n)=Q_{\text {down }} \\
Q_{u}(i)=\sum_{k=i}^{n} Q_{\text {out }}(k)+Q_{\text {down }} \\
-\sum_{k=i}^{n} q_{\text {lack }-t}(k)+\sum_{k=i}^{n} Q_{\text {sup }}(k) \\
Q_{d}(i)=Q_{u}(i+1)
\end{gathered}
$$

The above gate discharge is namely the input variables of discharge feed forward in the controller of canal system at the flow equilibrium regulation stage.

\section{E. Computational Example}

1) Basic Canal Conditions and Canal Reach Parameters

The emergency water supply canal of the middle route of China south-to-north water transfers project is selected as the study canal. The starting point is the ancient canal check gate of Shijiazhuang city, the end point is the canal endpoint of Hebei province segment. The original stake number is $968+909$, and the terminal stake number is $1196+167$, the overall length of the simulation canal is $227.4 \mathrm{~km}$. The entire canal system is divided into thirteen canal reaches by fourteen check gates; the design discharge is $170 \mathrm{~m}^{3} / \mathrm{s}$ at the starting point, and $60 \mathrm{~m}^{3} / \mathrm{s}$ at the end point. The canal system contains twelve canalside turnouts, sixteen inverted siphons, and three aqueducts, some under drains, culvert, bridges, transition sections and structures. The parameters of each canal pool see Table II .

In the study of this section, the upstream six canal reaches of the simulation canal is selected as the simulated object for simple ness (from canal pool 1 to canal pool 6 in Table II). Then the end stake number of the simulated object is $1070+382$, the overall length is $101.473 \mathrm{~km}$, the simulated object contains five canalside turnouts, six inverted siphons, one aqueduct, two under drains, one culvert and some transition sections and drainage structures.

Because there are variation conditions of cross section forms and so on within the canal reaches, so each canal reach needs to be divided into some sub-reaches again. The division of interior sub-reaches and the calculation parameters see [11] for details. The canal roughness coefficient $n=0.015$.

2) Parameters of the Inverted Siphons, Gates and Turnouts

The calculation methods and calculation coefficients of 
head loss of inverted siphons in canal system see [12]. Because lacks some relevant parameters of gates in initial data, the useful width of each gate is supposed to be $20 \mathrm{~m}$ referring to the connected structure width. The parameters of each turnout are shown in Table I .

TABLE I. PARAMETERS TABLE OF CANALSIDE TURNOUTS

\begin{tabular}{|c|c|c|c|}
\hline Turnout No. & Stake number & Position & Design discharge $\left(\mathrm{m}^{3} / \mathrm{s}\right)$ \\
\hline 1 & $982+418$ & pool 2 & 5 \\
\hline 2 & $1006+048$ & pool 3 & 2 \\
\hline 3 & $1029+321$ & pool 4 & 2 \\
\hline 4 & $1034+575$ & pool 4 & 20 \\
\hline 5 & $1059+925$ & pool 6 & 2 \\
\hline 6 & $1068+925$ & pool 6 & 3 \\
\hline 7 & $1102+941$ & pool 8 & 12 \\
\hline 8 & $1116+185$ & pool 9 & 0.5 \\
\hline 9 & $1120+325$ & pool 9 & 60 \\
\hline 10 & $1154+975$ & pool 11 & 2 \\
\hline 11 & $1179+272$ & pool 13 & 3 \\
\hline 12 & $1194+291$ & pool 13 & 11 \\
\hline
\end{tabular}

\section{3) Simulation Operating Condition and Results} Analysis

The discharge at the canal downstream end decreases linearly from $80 \mathrm{~m}^{3} / \mathrm{s}$ to $40 \mathrm{~m}^{3} / \mathrm{s}$ in $1-3$ hours, and the discharge at canalside turnouts decreases linearly from $100 \%$ design values to $40 \%$ design values in $1-3$ hours.
Supposes the water depth at the most upstream end is $7.0 \mathrm{~m}$ and maintains invariable. The initial condition of simulation is the steady flow state when the discharge at the canal downstream end and turnouts has not changed under the constant downstream depth operation.

The value of $R_{t}$ is 0.7 , the target water level $Y T_{\text {tar }}(i)$ is designed $0.3 \mathrm{~m}$ above the initial midpoint-weighted water level. Then according to the accurate control method of water volume of the controlled volume operation, the restricted water level $Z_{\max }(i)$ of beginning the flow equilibrium regulation can be acquired, see Table III. If any canal pool has arrived at its restricted water level, then the linear recovery regulation of discharge difference is conducted for $2 \mathrm{~h}$ for this canal pool to achieve the flow rate equilibrium between outflow and inflow. If all canal pools have achieved the flow rate equilibrium, then the water volume regulation of entire canal system is completed.

After the entire simulation process of the controlled volume operation is completed, the related characteristic parameters of volume variation of each canal pool are shown in Table III. The response processes of check gates see Fig. 5, the variation processes of gate discharge see Fig. 6, and the water level variation processes of part canal pools see Figs. 7-9. The control performance indicators [13] of canal operation see Table IV.

TABLE II. CHARACTERISTIC TABLE OF CANAL PARAMETERS

\begin{tabular}{|c|c|c|c|c|c|c|}
\hline \multirow{2}{*}{$\begin{array}{l}\text { Canal } \\
\text { pool No. }\end{array}$} & \multirow{2}{*}{$\begin{array}{l}\text { Number of inner } \\
\text { sub-reaches }\end{array}$} & \multirow{2}{*}{ Length (m) } & \multirow{2}{*}{$\begin{array}{l}\text { Design water } \\
\text { depth (m) }\end{array}$} & \multirow{2}{*}{$\begin{array}{c}\text { Design } \\
\text { discharge }\left(\mathrm{m}^{3} / \mathrm{s}\right)\end{array}$} & \multicolumn{2}{|c|}{ Bottom altitude (m) } \\
\hline & & & & & Upstream end & Downstream end \\
\hline 1 & 5 & 9759 & 6.00 & 170 & 70.253 & 70.141 \\
\hline 2 & 4 & 22053 & 5.00 & 170 & 69.991 & 69.032 \\
\hline 3 & 4 & 15177 & 5.00 & 165 & 68.882 & 67.728 \\
\hline 4 & 6 & 19553 & 5.00 & 155 & 67.578 & 66.475 \\
\hline 5 & 3 & 9234 & 5.00 & 135 & 66.325 & 66.139 \\
\hline 6 & 5 & 25697 & 4.50 & 135 & 65.989 & 64.935 \\
\hline 7 & 3 & 13198 & 4.50 & 135 & 64.812 & 64.295 \\
\hline 8 & 7 & 27098 & 4.50 & 135 & 64.145 & 61.486 \\
\hline 9 & 4 & 9717 & 4.50 & 125 & 61.336 & 60.771 \\
\hline 10 & 2 & 14924 & 4.50 & 100 & 60.621 & 59.920 \\
\hline 11 & 5 & 20829 & 4.30 & 60 & 59.770 & 58.695 \\
\hline 12 & 4 & 14705 & 4.30 & 60 & 58.545 & 57.849 \\
\hline 13 & 8 & 25314 & 4.30 & 60 & 57.699 & 56.500 \\
\hline
\end{tabular}

TABLE III. THE CHARACTERISTIC PARAMETERS OF VOLUME VARIATION OF EACH CANAL POOL

\begin{tabular}{|c|c|c|c|c|c|c|}
\hline Canal pool No. & Initial volume $\left(10^{6}\right)$ & $\begin{array}{c}\text { Steady } \\
\text { volume }\left(10^{6}\right)\end{array}$ & $\begin{array}{c}\text { Volume } \\
\text { variation }\left(10^{6}\right)\end{array}$ & $Z_{\max }(\mathrm{i})-Y T(\mathrm{i})$ & $\begin{array}{c}Y T_{\text {tar }}(\mathrm{i})-Y T(\mathrm{i}) \\
(\mathrm{m})\end{array}$ & $\begin{array}{c}q_{\text {lack }} \\
\left(\mathrm{m}^{3} / \mathrm{s}\right)\end{array}$ \\
\hline 1 & 1.23 & 1.34 & 0.10 & 0.264 & -3.63 \\
\hline 2 & 3.48 & 3.79 & 0.31 & 0.265 & 0.298 & -10.25 \\
\hline 3 & 2.16 & 2.35 & 0.19 & 0.265 & -6.302 \\
\hline 4 & 2.98 & 3.25 & 0.27 & 0.263 & -8.79 \\
\hline
\end{tabular}




\begin{tabular}{|l|l|l|l|l|l|l|}
\hline 5 & 1.19 & 1.30 & 0.11 & 0.267 & 0.298 & -3.50 \\
\hline 6 & 3.45 & 3.81 & 0.35 & 0.268 & 0.299 & -10.18 \\
\hline
\end{tabular}

(Note: YT(i) is the initial midpoint-weighted water level of each canal pool)

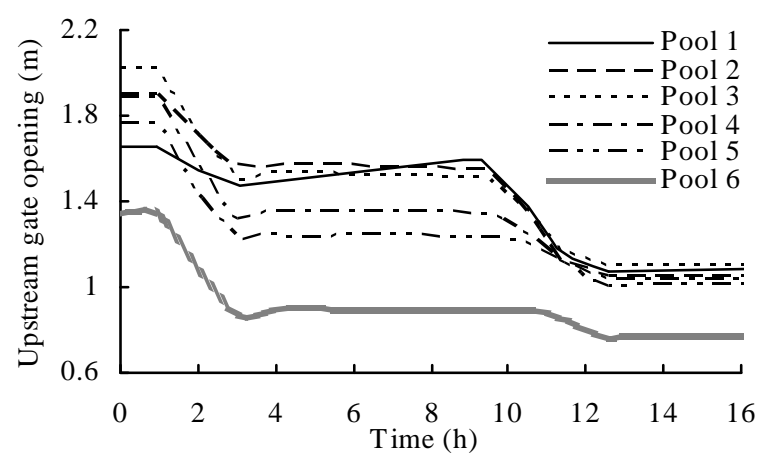

Figure 5. Upstream gate opening processes of each canal pool

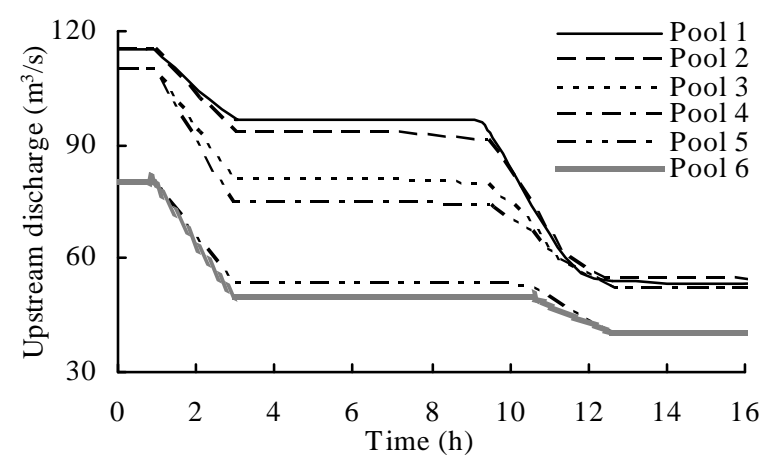

Figure 6. Upstream gate discharge processes of each canal pool

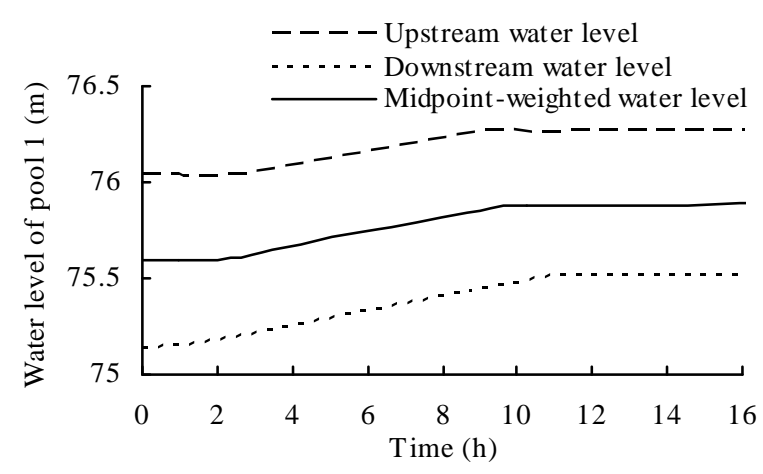

Figure 7. Water level variation processes of canal pool 1

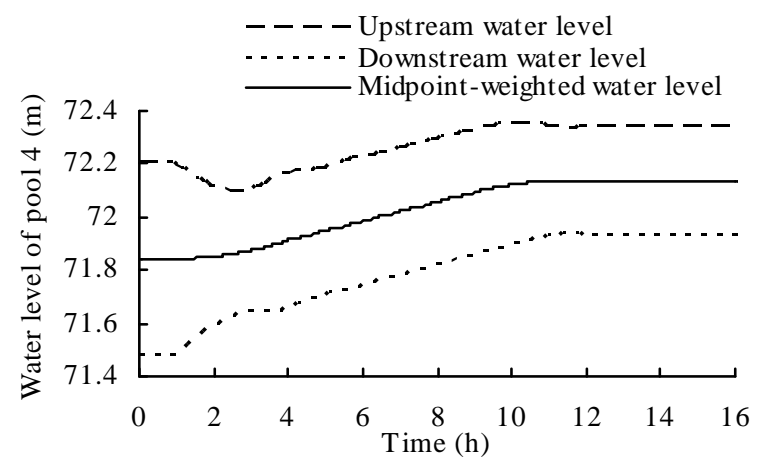

Copyright (C) 2009 MECS
Figure 8. Water level variation processes of canal pool 4

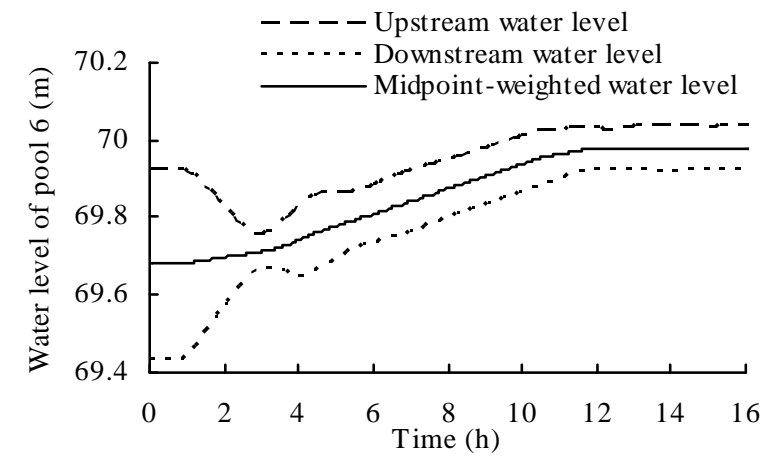

Figure 9. Water level variation processes of canal pool 6

TABLE IV. CONTROL PERFORMANCE INDICATORS

\begin{tabular}{|c|c|c|c|c|}
\hline $\begin{array}{c}\text { Canal pool } \\
\text { No. }\end{array}$ & $\begin{array}{c}M A E \\
(\%)\end{array}$ & $\begin{array}{c}I A Q \\
\left(\mathrm{~m}^{3} / \mathrm{s}\right)\end{array}$ & $\begin{array}{c}I A W \\
(\mathrm{~m})\end{array}$ & $\begin{array}{c}I S E \\
\left(10^{-7} \mathrm{~m}\right)\end{array}$ \\
\hline 1 & 0.059 & 0.015 & 0.257 & 4.63 \\
\hline 2 & 0.128 & 0.191 & 0.052 & 7.81 \\
\hline 3 & 0.069 & 0.105 & 0.104 & 3.40 \\
\hline 4 & 0.117 & 0.088 & 0.110 & 3.69 \\
\hline 5 & 0.069 & 0.128 & 0.101 & 5.69 \\
\hline 6 & 0.296 & 0.314 & 0.087 & 30.96 \\
\hline
\end{tabular}

The simulation results indicate:

1) When the discharge demand at the canal downstream end and canalside turnouts decreases, in the first round regulation of check gates, uses the disequilibrium between outflow and inflow of canal pool to supply storage volume, and the water level of each canal pool ascends. The second round regulation of check gates makes the outflow and inflow of canal pool to be balanceable, and the water level tends to be stable.

2) The water level indicators MAE and ISE of canal operation are relatively ideal, which indicates the system has good control effect over the water level. The values of subordinate performance indicators IAQ and $I A W$ are very small, which indicates the processes of gate discharge and gate opening are also ideal in the process of system operation, and the gates have nearly not alternate motion.

3) From the variation processes of gate opening we can see, the gate opening of the head of canal still has relatively obvious variation in the stable flow delivery stage when the absolute discharge difference has reached its maximum value (approximately in 3-9h). This is because the upstream water level of the head gate maintains constant, but the downstream water level of the head gate rises gradually along with the increase of water volume, therefore, the gate opening needs to increase gradually to maintain stable gate discharge.

4) The canal system of the controlled volume method of operation proposed in the paper has very high 
flexibility of operation and control. Through multi-rounds regulation, the water volume is rationally distributed according to proportion in time domain, it has guaranteed each canal pool to be effectively controlled regardless of the length of canal pool or participation of canalside turnouts. The midpoint-weighted water levels of all canal pools vary smoothly, the amplitude of water level fluctuation of upstream and downstream ends is also small. Moreover, the controlled volume algorithm proposed in the paper can comparatively accurately control the water volume or target water level of each canal pool (the absolute error of the stable target water level of each canal pool is less than or equal to $0.002 \mathrm{~m}$ ), therefore, the adjustable water volume of each canal pool is fully used.

\section{Algorithm design of the Controlled Volume Operation Method of ContinuOUs CANAL PoOlS}

When the downstream continuous canal pools of canal system have no discharge demand change, and only some turnouts between canal head and middle canal pool of system have discharge demand change, if we adopt the controlled volume operation of whole canal system, it certainly will cause some unnecessary regulation, and consequently increases disturbed canal pools.

Therefore, when the discharge demand at some most downstream canal pools is invariable, and only some canalside turnouts between canal head and certain middle pool have discharge variation, then the most downstream pool which has discharge variation can be treated as an interior boundary, and the controlled volume regulation is made to this pool as well as all its upstream pools, all downstream pools of this pool can keep their original operation state and be invariable. When the most downstream discharge variation pool is closer to canal head, the advantage of this regulation method is more conspicuous. Therefore, the operation algorithm proposed is based on the assumption that some most downstream pools have no discharge variation, and when all canal pools of system have discharge variation, this algorithm can also be used directly.

\section{A. Regulation of discharge difference}

Suppose the most downstream canal pool in which the discharge demand changes is the pool $l$ (Fig. 10).

The calculation methods of $Q_{\text {con }}, Q_{\text {sum }}$ and $Q_{\text {lack }}$ are the same as above paragraph.

The discharge difference between the inflow and outflow in each canal pool is:

$$
\begin{array}{cc}
\text { If } \quad l<i \leq n & q_{\text {lack }}^{j}(i)=0 \\
\text { If } & 1 \leq i \leq l \quad q_{\text {lack }}^{j}(i)=R_{t} \times Q_{\text {lack }}^{j} \times \frac{V^{j}(i)}{\sum_{i=1}^{n} V^{j}(i)}
\end{array}
$$

Where the meanings of all symbols are the same as above paragraph.

From the downstream end pool $n$ to the upstream pool $i(i=n-1, n-2, \ldots .2,1)$, the upstream and downstream gate discharge of each pool are in turn:

$$
\text { If } l<i \leq n \text { : }
$$

$$
\begin{gathered}
Q_{u}(i)=\sum_{k=i}^{n} Q_{o}(k)+Q_{\text {down }} \\
Q_{d}(i-1)=Q_{u}(i) \\
Q_{d}(n)=Q_{\text {down }}
\end{gathered}
$$

If $1 \leq i \leq l$ :

$$
\begin{aligned}
& Q_{u}(i)=\sum_{k=i}^{l} Q_{\text {out }}(k)+ \\
& \sum_{k=l+1}^{n} Q_{o}(k)+Q_{\text {down }}-\sum_{k=i}^{l} q_{\text {lack }}(k) \\
& Q_{d}(i-1)=Q_{u}(i)
\end{aligned}
$$

\section{B. Flow Equilibrium regulation algorithm}

At the discharge equilibrium regulation stage, from the downstream end pool $n$ to upstream pool $i$, the upstream and downstream gate discharge of each pool are:

$$
\begin{gathered}
\text { If } \begin{array}{c}
l<i \leq n: \\
Q_{u}(i)=\sum_{k=i}^{n} Q_{o}(k)+Q_{\text {down }} \\
Q_{d}(i-1)=Q_{u}(i) \\
Q_{d}(n)=Q_{\text {down }} \\
\text { If } \quad 1 \leq i \leq l: \\
Q_{u}(i)=\sum_{k=i}^{l} Q_{\text {out }}(k)+\sum_{k=l+1}^{n} Q_{o}(k)+Q_{\text {down }}- \\
\sum_{k=i}^{l} q_{\text {lack-t }}(k)+\sum_{k=i}^{l} Q_{\text {sup }}(k) \\
Q_{d}(i-1)=Q_{u}(i)
\end{array}
\end{gathered}
$$

Where the meanings of all symbols are the same as the former.

The upstream and downstream discharge of each pool derived from the controlled volume algorithm are namely the input variables of the discharge feed forward control.

The real-time target point water level calculation, and the determination of the target water level and restricted water level see above paragraph.

\section{Computational Example}

The thirteen canal reaches in the emergency water supply canal of the middle route of China south-to-north water transfers project is selected as the simulation canal. The overall parameters of simulated canal see Tables I and II.

The discharge at the canal downstream end is $30 \mathrm{~m}^{3} / \mathrm{s}$, and keeps invariable, the discharge at the Zhong Guantou turnout (belonging to canal pool 4 ) increases linearly from $5 \mathrm{~m}^{3} / \mathrm{s}$ to $20 \mathrm{~m}^{3} / \mathrm{s}$ in $1-5$ hours, and other turnouts have no water intake discharge.

In the process of canal operation, only canal pool 1, 2, 3 and 4 participate in storage volume regulation, other pools keep their original operating state invariable. 
The target water level $Y T_{\text {tar }}(i)$ of canal pools with storage volume regulation are designed $0.15 \mathrm{~m}$ below their initial midpoint-weighted water levels. Then according to the accurate control method of water volume, the restricted water level $Z_{\text {min }}(i)$ can be acquired. If any pool has arrived at its restricted water level, then the linear recovery regulation of discharge difference is conducted for $2 \mathrm{~h}$ for this pool to balance the final pool inflow and outflow. If all pools have achieved the flow rate equilibrium, then the water volume regulation of entire canal system is completed. The value of $R_{t}$ is 0.7 .

After the entire simulation process is completed, the related characteristic parameters of volume variation are shown in Table $\mathrm{V}$. The response processes of check gates under the controlled volume operation of continuous canal pools and the controlled volume operation of whole canal system are shown in Fig. 11 and Fig. 12, respectively. The variation processes of gate discharge see Fig. 13, and the water level variation processes of part pools see Figs. 14-15. The performance indicators [13] of canal operation see Table VI.

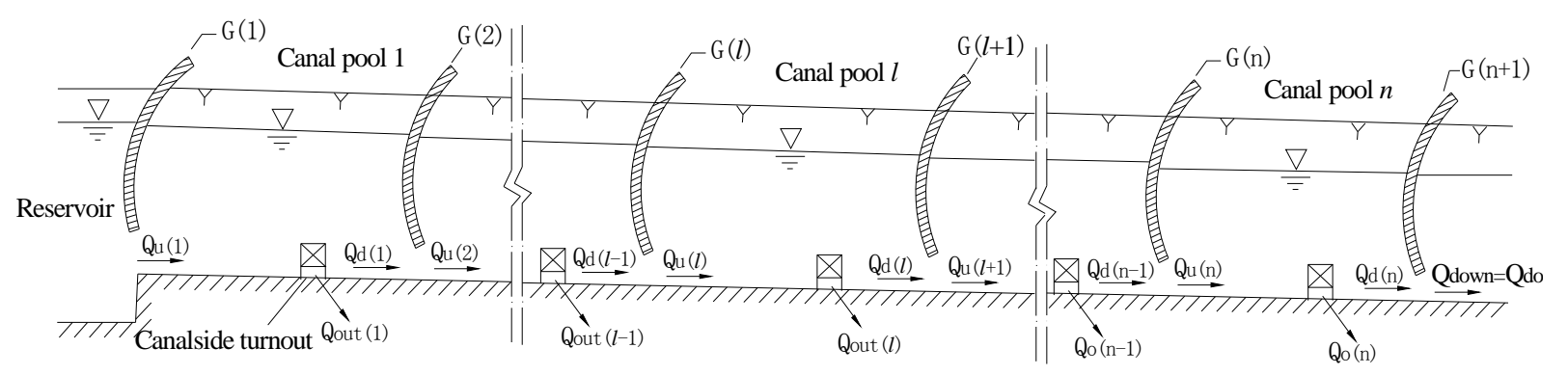

Figure 10. Sketch map of canal system under controlled volume operation of continuous canal pools

TABLE V. THE CHARACTERISTIC PARAMETERS OF VOLUME VARIATION OF EACH CANAL POOL

\begin{tabular}{|c|c|c|c|c|c|c|}
\hline $\begin{array}{l}\text { Canal pool } \\
\text { No. }\end{array}$ & $\begin{array}{c}\text { Initial } \\
\text { volume } \\
\left(10^{6} \mathrm{~m}^{3}\right)\end{array}$ & $\begin{array}{l}\text { Steady volume } \\
\qquad\left(10^{6} \mathrm{~m}^{3}\right)\end{array}$ & $\begin{array}{l}\text { Volume variation } \\
\qquad\left(10^{6} \mathrm{~m}^{3}\right)\end{array}$ & $\begin{array}{l}\text { Initial target point } \\
\text { water level (m) }\end{array}$ & $\begin{array}{l}\text { Steady target point } \\
\text { water level (m) }\end{array}$ & $\begin{array}{c}\text { Variation of target } \\
\text { point water level (m) }\end{array}$ \\
\hline 1 & 1.209 & 1.159 & -0.050 & 75.515 & 75.364 & -0.151 \\
\hline 2 & 3.295 & 3.149 & -0.146 & 74.106 & 73.958 & -0.148 \\
\hline 3 & 2.090 & 2.000 & -0.091 & 73.058 & 72.908 & -0.150 \\
\hline 4 & 2.853 & 2.726 & -0.127 & 71.672 & 71.521 & -0.151 \\
\hline 5 & 1.170 & 1.170 & 0 & 70.827 & 70.827 & 0 \\
\hline 6 & 3.263 & 3.263 & 0 & 69.488 & 69.488 & 0 \\
\hline 7 & 1.767 & 1.767 & 0 & 68.811 & 68.811 & 0 \\
\hline 8 & 3.240 & 3.240 & 0 & 66.821 & 66.821 & 0 \\
\hline 9 & 1.358 & 1.358 & 0 & 65.306 & 65.306 & 0 \\
\hline 10 & 0.909 & 0.909 & 0 & 64.474 & 64.474 & 0 \\
\hline 11 & 1.389 & 1.389 & 0 & 63.262 & 63.262 & 0 \\
\hline 12 & 1.001 & 1.001 & 0 & 62.301 & 62.301 & 0 \\
\hline 13 & 1.594 & 1.594 & 0 & 60.841 & 60.841 & 0 \\
\hline
\end{tabular}

TABLE VI. CONTROL PERFORMANCE INDICATORS

\begin{tabular}{|c|c|c|c|c|c|c|c|c|}
\hline \multirow{2}{*}{$\begin{array}{c}\text { Canal } \\
\text { pool } \\
\text { No. }\end{array}$} & \multicolumn{4}{|c|}{ Controlled volume operation of continuous canal pools } & \multicolumn{4}{|c|}{ Controlled volume operation of whole canal pools } \\
\hline & $\begin{array}{c}\text { MAE } \\
(\%)\end{array}$ & $\begin{array}{c}I A Q \\
\left(\mathrm{~m}^{3} / \mathrm{s}\right)\end{array}$ & $\begin{array}{c}\text { IAW } \\
(\mathrm{m})\end{array}$ & $\begin{array}{c}I S E \\
\left(10^{-8} \mathrm{~m}\right)\end{array}$ & $\begin{array}{c}M A E \\
(\%)\end{array}$ & $\begin{array}{c}I A Q \\
\left(\mathrm{~m}^{3} / \mathrm{s}\right)\end{array}$ & $\begin{array}{l}I A W \\
(\mathrm{~m})\end{array}$ & $\begin{array}{c}I S E \\
\left(10^{-8} \mathrm{~m}\right)\end{array}$ \\
\hline 1 & 0.023 & 0.008 & 0.056 & 9.90 & 0.009 & 0.006 & 0.016 & 1.53 \\
\hline 2 & 0.019 & 0.015 & 0.020 & 1.25 & 0.012 & 0.012 & 0.008 & 1.70 \\
\hline 3 & 0.024 & 0.044 & 0.036 & 6.45 & 0.010 & 0.019 & 0.015 & 1.18 \\
\hline 4 & 0.013 & 0.018 & 0.024 & 0.93 & 0.008 & 0.015 & 0.011 & 0.90 \\
\hline 5 & 0 & 0.001 & 0.004 & 0. & 0.008 & 13.122 & 0.265 & 1.02 \\
\hline 6 & 0 & 0.001 & 0 & 0 & 0.022 & 12.132 & 0.181 & 5.57 \\
\hline 7 & 0 & 0.001 & 0 & 0 & 0.008 & 9.415 & 0.183 & 0.66 \\
\hline 8 & 0 & 0.001 & 0 & 0 & 0.014 & 7.940 & 0.116 & 2.42 \\
\hline 9 & 0 & 0.002 & 0 & 0 & 0.011 & 5.238 & 0.106 & 1.41 \\
\hline 10 & 0 & 0.001 & 0 & 0 & 0.014 & 4.094 & 0.091 & 0.56 \\
\hline 11 & 0 & 0.001 & 0 & 0 & 0.015 & 3.335 & 0.075 & 2.76 \\
\hline
\end{tabular}




\begin{tabular}{|l|l|l|l|l|l|l|l|l|}
12 & 0 & 0.001 & 0 & 0 & 0.015 & 2.175 & 0.057 & 2.82 \\
\hline 13 & 0 & 0.001 & 0 & 0 & 0.031 & 1.320 & 0.028 & 11.88 \\
\hline
\end{tabular}

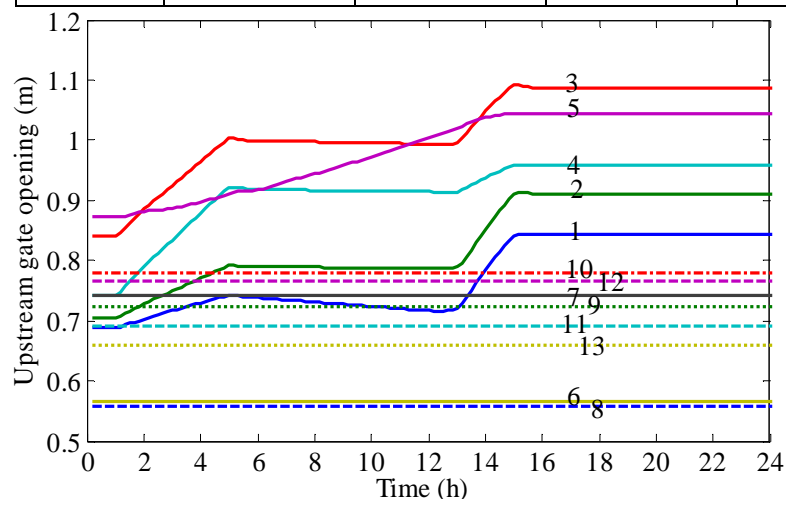

Figure 11. Upstream gate opening processes under the controlled volume operation of continuous canal pools

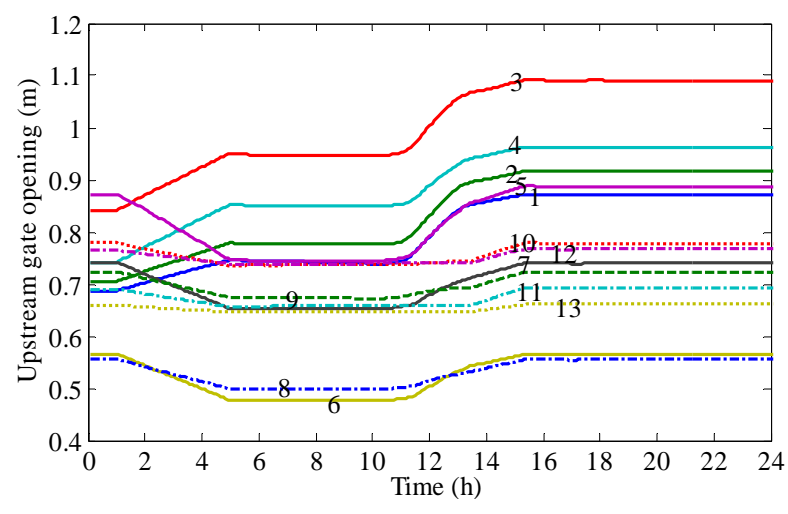

Figure 12. Upstream gate opening processes under the controlled volume operation of whole canal system

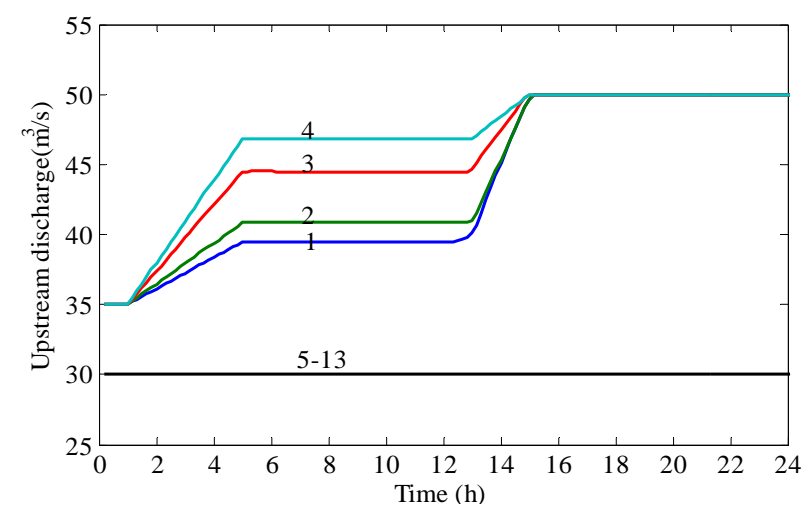

Figure 13. Upstream gate discharge processes

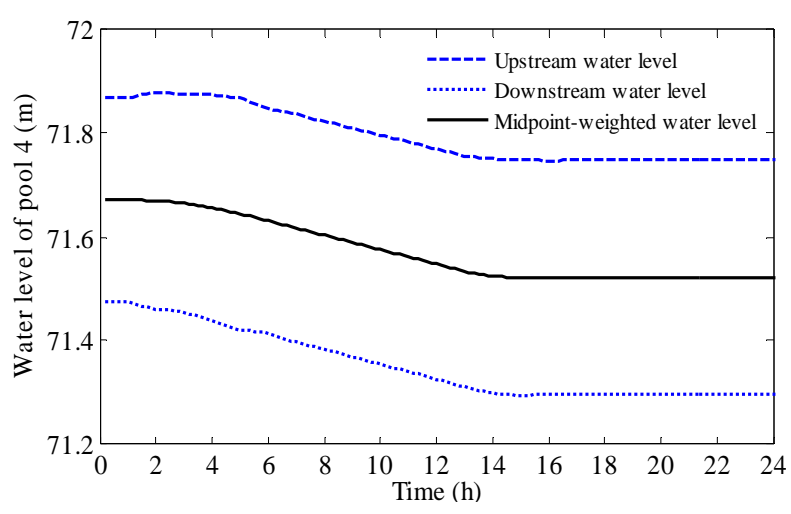

Figure 14. Water level variation processes of pool 4

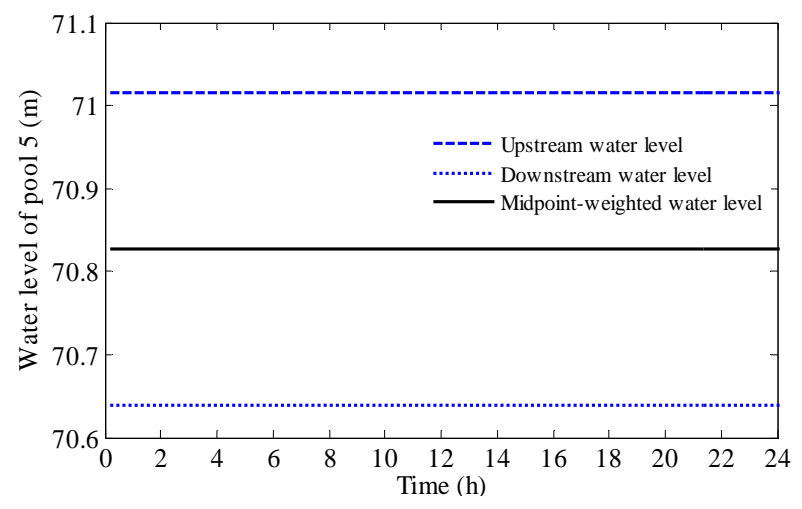

Figure 15. Water level variation processes of pool 5

The simulation results indicate:

1) When some downstream continuous canal pools have no demand change, the controlled volume algorithm proposed in the paper can realize controlled volume operation of continuous canal pools. The downstream continuous canal pools in which there is no discharge demand variation don't participate in the water volume regulation, and keep their original operating state invariable, so the regulated check gates and disturbed canal pools are decreased, and the downstream continuous canal pools keep balanced water supply all the while. Especially, the closer of the most downstream discharge demand change canal pool is to the head of canal, namely the closer of $l$ is to 1 , the better control effect of this algorithm is.

2) Because the most downstream turnout in which discharge demand changes belongs to canal pool 4, so canal pool 1, 2, 3 and 4 participated in controlled volume operation, and gate 1, 2, 3 and 4 are regulated according to the controlled volume algorithm. In order to guarantee constant discharge delivery, the gate 5 also should be adjusted according to its upstream and downstream water levels. The water level and discharge transition processes in regulated canal pools are relatively good, the same as the transition processes of regulated gates, so the control effect is relatively good. Because there is no discharge demand change from pool 5 to pool 13, they keep initial operating states invariable, so the number of disturbed canal pools and regulated gates is decreased.

3) All performance indicators of regulated canal pools are slightly larger than the controlled volume operation of whole canal system, it is because that the target point water level variation is relatively larger under the controlled volume operation of continuous canal pools, and all performance indicators of other pools under this operation method is equal to or close to zero. The indicators $I A W$ and $I A Q$ of canal pools 5 to 13 under the controlled volume operation of whole canal system have relatively larger increase than pools 1 to 4 , the reason for this is that the gates have reciprocating movement in pools 5 to 13 , and discharge has certain 
overshoot. All in all, the control effect of the controlled volume operation of continuous canal pools is better.

4) Under the same operating conditions, the canal response time of the controlled volume operation of continuous canal pools is shorter than the controlled volume operation of whole canal system, it's because the total discharge difference is distributed among lesser canal pools under the controlled volume operation of continuous canal pools, so the distributive discharge difference of each pool is relatively larger, and the time needed to accomplish the target storage volume regulation is shorter.

\section{CONCLUSION}

The controlled volume method of operation is a more flexible canal operation method. This method of operation can fully use the adjustable water volume of canal; the canal water volume can be increased or decreased to buffer the rapid flow change to enhance the stability of canal operation. An operating simulation mode based on storage volume control method for multireach canal system in series under gate regulating is established in the paper. The algorithm of the controlled volume operation of whole canal pools was proposed, and the simulation to practical project indicates this method of operation can rapidly respond to discharge change of the canal downstream end and canalside turnouts, and can comparatively accurately control the water volume change. The variation of gate opening and water levels is comparatively smooth in the process of system operation, the control performance indicators of canal operation are very good; Furthermore, in allusion to some typical operating conditions, the algorithm of the controlled volume operation of continuous canal pools is proposed, the simulation to practical project indicates the new algorithm can relatively obviously reduce the number of regulated gates and disturbed canal pools, the control effect of canal system can be obviously improved. The controlled volume method of operation is especially suitable for long distance water transfer project, which lacks on-line regulation reservoirs but has larger canal pool storage. However, the controlled volume method of operation proposed in the paper has acquired good control effects only in non-ice period operation conditions. Under the dispatching operation of the ice period water delivery, the controlled volume method of operation needs to be made further study.

\section{ACKNOWLEDGMENT}

The study is financially supported by the Key Projects in the National Science \& Technology Pillar Program during the 11th Five-Year Plan Period of China (Grant No: 2006BAB04A12).

\section{REFERENCES}

[1] C. P. Buyalski, D. G. Ehler, H. T. Falvey, D. C. Rogers, and E. A. Serfozo, "Canal systems automation manual,
Vol.1,” Denver: U. S. Department of the Interior, Bureau of Reclamation, 1991.

[2] H. G. Dewey and W. B. Madsen, "Flow control and transients in the California aqueduct," Journal of the Irrigation and Drainage Division, ASCE, vol.102, No.IR3, pp.335-348, September 1976.

[3] Roger, D. C. Coeuret and J. Bremond, "Dynamic regulation on the canal de provence,” Planning, Operation, Rehabilitation, and Automation of Irrigation Water Delivery Systems, ASCE, edited by D.D.Zimbelman, pp.180-200, New York, 1987.

[4] L. Becker, A. L. Graves, and W. W-G. Yeh, "Central Arizona project operation,” Proceedings of the International Symposium on Rainfall-Runoff Modeling, Water Resources Publication, Littleton, Colorado, 1981.

[5] D. C. Roger, "Automation of the canal de Cartagena, Spain,” Water and Wastewater International, vol.5, Issue 2, pp.35-40, Houston, Texas, Apr. 1990.

[6] Xiong Yao, Changde Wang, and Changjing Li, “Operation model of serial canal system based on water volume control method," ShuiLi XueBao, vol. 39, no. 6, pp. 733-738, Jun. 2008.

[7] Yao Xiong, "Research on automatic operation and control of long distance water transfer canal system," Ph.D. Thesis, Wuhan: Wuhan University, Apr. 2008.

[8] T.S. Strelkoff and H.T. Falvey, "Numerical method used to model unsteady canal flow," Journal of Irrigation and Drainage Engineering, vol. 119, no. 4, pp. 637-655, Aug. 1993.

[9] Guangming Tan, Zhiliang Ding, Changde Wang, and Xiong Yao, "Gate regulation speed and transition process of unsteady flow in channel," Journal of Hydrodynamics, vol. 20, no.2, pp. 231-238, May 2008.

[10] Fubo Liu, Jan Feyen, and Jean Berlamont, "Downstream control algorithm for irrigation canals," Journal of Irrigation and Drainage Engineering, vol. 120, no. 3, pp. 468-483, Jun. 1994.

[11] Fan Jie, "Characteristis analysis of canal operation and research of intelligent control," Ph.D. Thesis, Wuhan: Wuhan University, Apr. 2006.

[12] Zhiliang Ding, Changde Wang, Guangming Tan, and Guanghua Guan, "Processing method of the head loss of structures in large-scale water delivery channel system," South-to-North Water Transfers and Water Science \& Technology, vol. 6, no. 5, pp. 18-23, Oct. 2008.

[13] A. J. Clemmens, T. F. Kacerek, B. Grawitz, and W. Schuurmans, "Test cases for canal control algorithms," Journal of Irrigation and Drainage Engineering, vol. 124, no. 1, pp. 23-30, Feb. 1998.

Ding Zhiliang was born in Xinyang city, Henan province, China, in 1981. He received Ph.D. degree in water conservancy engineering from Wuhan University, China in 2009.

He is currently an engineer with the Changjiang Institute of Survey, Planning, Design and Research, Wuhan, China. His research interest includes canal system automatization and river flood control planning, et al.

Wang Changde was born in Xi'an city, Shanxi province, China. He received the master degree in water conservancy and hydropower engineering from Wuhan University, China in 1980.

He is currently a professor with the College of Water Conservancy and Hydropower, Wuhan University, Wuhan, China. His research interest includes canal system automatization and hydraulic structure, et al. 